\title{
A novel four-snoRNA signature for predicting the survival of patients with uveal melanoma
}

\author{
QIONG YI and WEN-JIN ZOU \\ Department of Ophthalmology, The First Affiliated Hospital of Guangxi Medical University, Nanning, \\ Guangxi Zhuang Autonomous Region 530021, P.R. China
}

Received July 6, 2018; Accepted November 14, 2018

DOI: $10.3892 / \mathrm{mmr} .2018 .9766$

\begin{abstract}
Uveal melanoma (UM), the predominant histological subtype of intraocular malignant tumors in adults, often results in high rates of mortality; effective prognostic signatures used to predict the survival of patients with UM are limited. Small nucleolar RNAs (snoRNAs) are emerging as important regulators in the processes of carcinogenesis and tumor progression, but knowledge of their application as prognostic markers in UM is limited. In the present study, the expression profiles of snoRNAs in UM were determined; a total of 60 snoRNAs were notably associated with the overall survival of patients with UM via univariate Cox survival analysis. Subsequently, a prognostic signature based on four snoRNAs was proposed, which retained their prognostic significance determined by a multivariate Cox survival analysis. The formula is as follows: ACA17 * (-1.602) + ACA45 *0.803 + HBII-276 * $0.603+$ SNORD12 $* 1.348$. Furthermore, the results of in silico analysis indicated that perturbation of the phototransduction, GABAergic synapse and amphetamine addiction pathways may be the potential molecular mechanisms underlying the poor prognosis of patients with UM. Collectively, the present study proposed a potential prognostic signature for patients with UM and the prospective mechanisms at the genome-wide level were determined.
\end{abstract}

\section{Introduction}

Uveal melanoma (UM), a rare subset of melanoma accounting for only 5\% of all melanomas, makes up 85-95\% of all ocular melanomas; however, UM ranks as the predominant intraocular malignancy in adult patients (1-4). Tumors located on the choroid

Correspondence to: Dr Wen-Jin Zou, Department of Ophthalmology, The First Affiliated Hospital of Guangxi Medical University, 6 Shuangyong Road, Nanning, Guangxi Zhuang Autonomous Region 530021, P.R. China

E-mail: bigstone168@163.com

Key words: uveal melanoma, small nucleolar RNAs, precision medicine, prognostic signature, clinical outcome are the most common, affecting $\leq 90 \%$ of patients (5-7). In addition, $\sim 50 \%$ of patients will develop metastasis, $>90 \%$ of which disseminates to the liver $(8,9)$. Once the tumor has metastasized, the median overall survival (OS) is only 6-12 months (10-12). The average age at diagnosis is 60-years-old, and UM affects both sexes almost equally, with a slightly increased frequency in males $(13,14)$. Recent advances in the detection of molecular pathology and a various immune-based therapies have exhibited efficacy in treating UM. However, the molecular biomarkers that may be effective in predicting the development and prognosis of UM in patients are limited, which has led to the unsatisfactory clinical management and targeted therapy of this disease (15-17); thus, UM is associated with high mortality. Therefore, there is an urgent need to identify ideal prognostic biomarkers and treatment options for patients with UM.

Recently, increasing evidence has indicated that non-coding RNAs serve a vital regulatory role in tumorigenesis and tumor progression, including small nucleolar RNAs (snoRNAs) $(18,19)$. snoRNAs, encoded by introns, exist stably in the cellular environment and can regulate the expression of specific genes (20-23). Numerous studies have proposed that snoRNA expression profiles are dysregulated in various types of tumors $(24,25)$. Furthermore, numerous dysregulated snoRNAs have also been identified to be associated with the development and prognosis of cancer, including melanoma, which suggests the potential of snoRNAs as prognostic predictors (26-28). Unfortunately, the association between snoRNAs and UM has rarely been reported.

In the present study, the expression landscape of snoRNAs was integrated using the snoRNA in cancers (SNORic) database and the clinical information of patients with UM in The Cancer Genome Atlas (TCGA) database. snoRNas associated with survival were systematically selected and a specific prognosis index (PI) was proposed, which could be an ideal prognostic signature to predict the prognosis of patients with UM. Further bioinformatics analysis revealed survival-associated pathways in UM. These findings may aid the identification of the novel biological functions of snoRNAs in UM, and improve the clinical management of this disease.

\section{Materials and methods}

General characteristics. The data of 80 patients with UM were submitted for survival analysis. Among the 80 patients with 


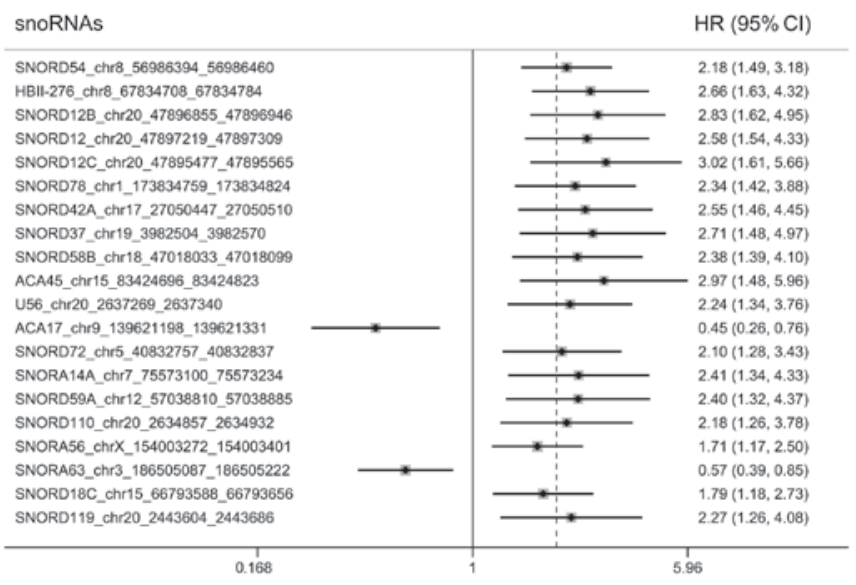

Figure 1. The top 20 most significant survival-associated small nucleolar RNAs. HR, hazard ratio.

UM, the histological types included epithelioid cell $(n=34)$ and spindle cell $(n=46)$. A total of 35 patients were female and 45 patients were male. The average age was 61.65 years (ranging from 22-86 years).

Data acquisition. SnoRNA expression profiles were downloaded from the SNORic (http://bioinfo.life.hust.edu. $\mathrm{cn} / \mathrm{SNORic}$ ) database, which provides the quantified expression levels of snoRNAs in the form of reads per kilobase per million (RPKM). Then, the original values were calculated via $\log 2$ conversion. The corresponding clinical information of UM patients was also downloaded from the TCGA data portal (http://cancergenome.nih.gov/). To obtain more accurate results, low-abundance snoRNAs with average log (RPKM+1) expression levels in all samples were omitted.

Survival analysis. A univariate Cox regression analysis was performed to select snoRNAs that were correlated with the overall survival (OS) of patients with UM. Subsequently, multivariate Cox proportional hazards regression was implemented to further identify whether the prognostic snoRNAs were independent biomarkers. Finally, the snoRNA-based prognostic signature was proposed according to the linear multiplication of the expression profiles of each prognostic snoRNA, weighted by their estimated regression coefficients in the multivariate Cox analysis. All survival analyses were conducted using the Survival package version 2.43-1 in R software version 3.4.4 (https://CRAN.R-project. org/package=survival). Then, patients were separated into high- and low-risk groups according to median risk score values. Kaplan-Meier (K-M) survival analysis followed by a log-rank test was used to assess the value of the snoRNAs in predicting prognosis. The number of patients in the low- and high-risk groups was recorded every 500 days.

Bioinformatics procedure. To further investigate the potential pathways affected in the high- and low-risk groups, the Limma package version 3.38.2 in R software was used to identify the differentially expressed genes between high- and low-risk groups (29). The mRNA expression profiles were downloaded from TCGA database; the raw count data of mRNAs were submitted for analysis. The thresholds for definite

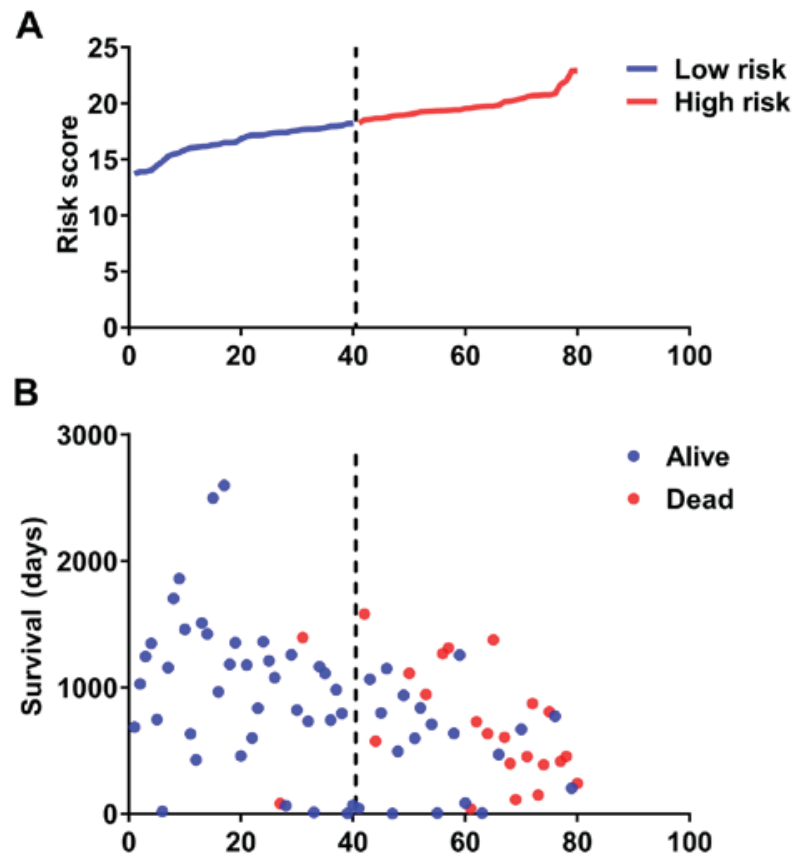

Figure 2. Small nucleolar RNA-based risk scores of patients with UM. (A) Lowand high-risk group snoRNA signatures in patients with UM. (B) Survival status and duration of survival of patients with UM. UM, uveal melanoma.
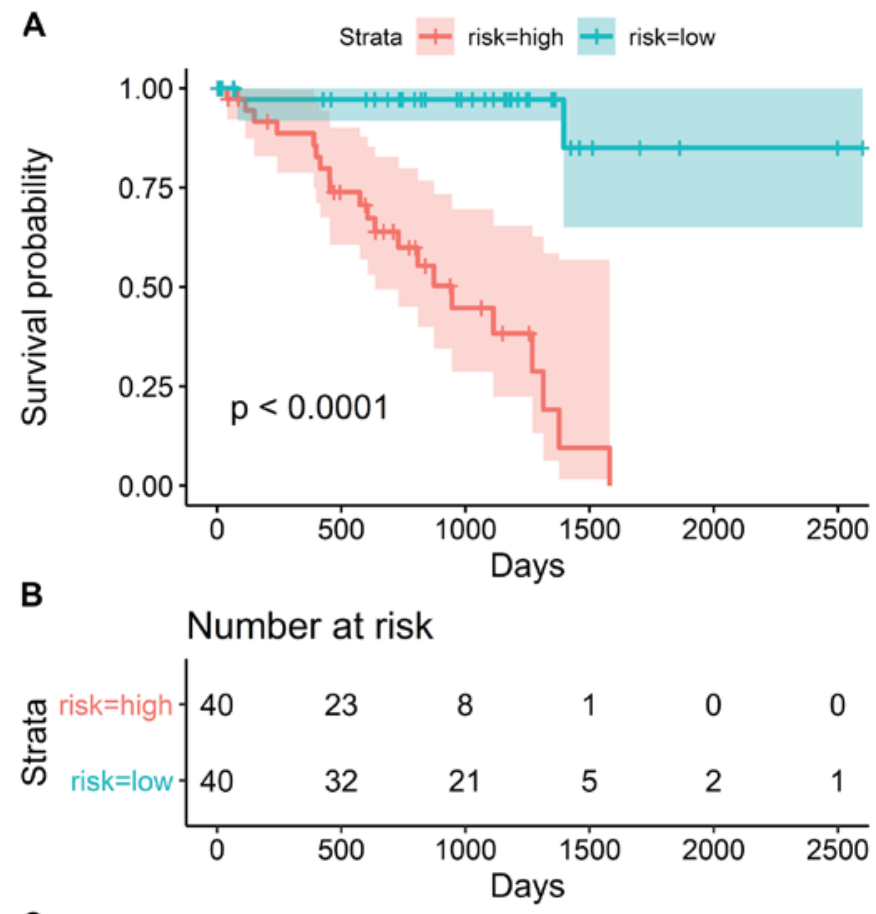

C

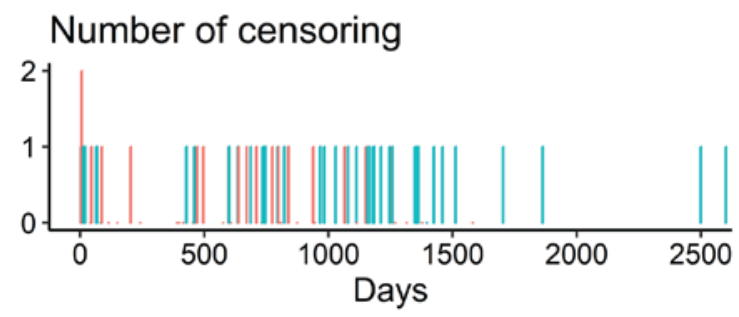

Figure 3. Patients in the low- and high-risk groups exhibit distinct clinical outcomes. (A) Kaplan-Meier analysis revealed that patients in the high-risk group exhibited poorer overall survival than patients in the low-risk group. (B) The number of patients in the low- and high-risk groups at various time-points. (C) The number of censored cases at various time-points. 
A

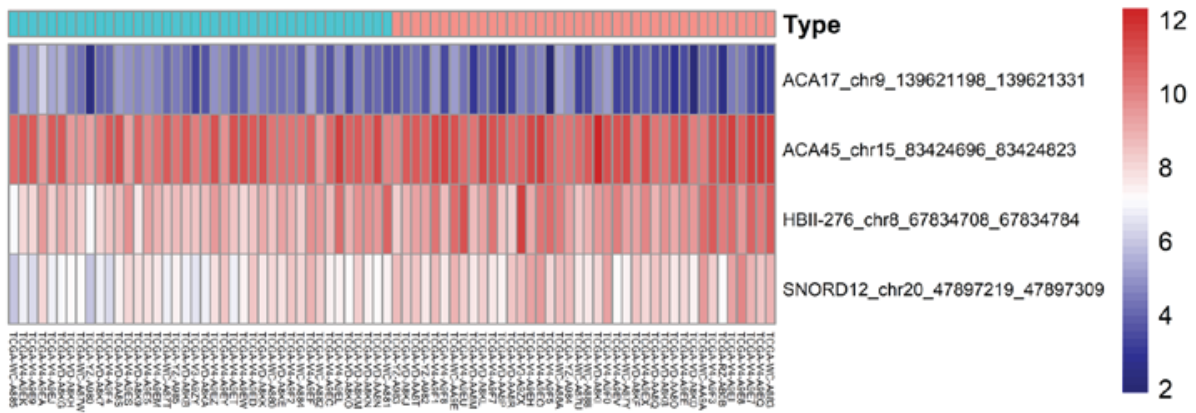

B

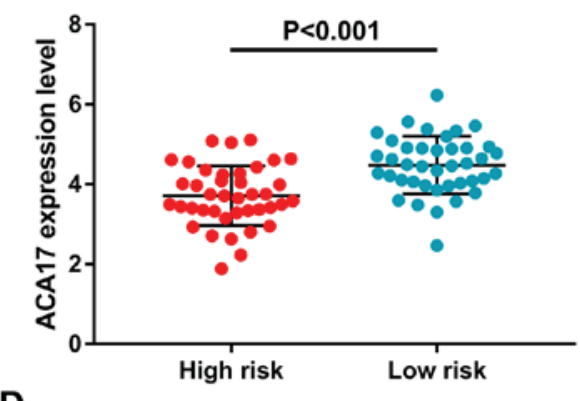

D

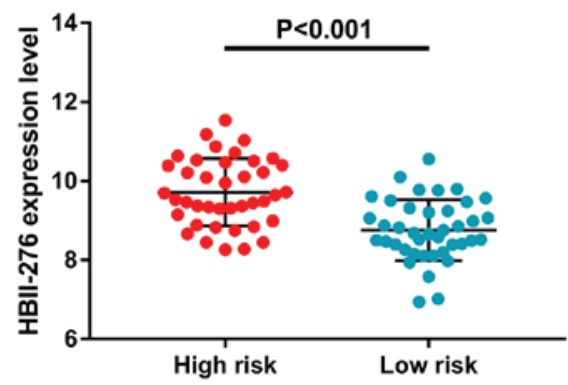

C

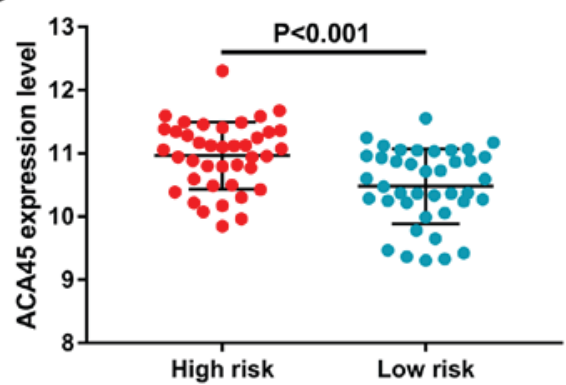

E

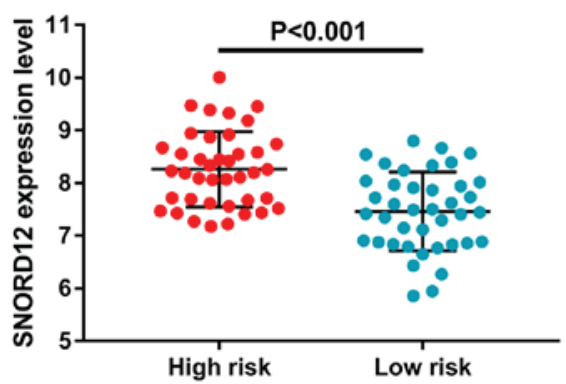

Figure 4. Expression of the four small nucleolar RNAs in the high- and low-risk groups. (A) Heatmap of the expression of the four key snoRNAs in uveal melanoma. The colors from blue to red indicate the trend from low expression to high expression. (B) ACA17 was downregulated in the high-risk group, and (C) ACA45, (D) HBII-276 and (E) SNORD12 were upregulated in high-risk group.

differentially expressed genes were set to llog fold changel $>2$ and false discovery rate $<0.01$. Subsequently, differentially expressed genes were submitted to the DAVID online database for gene ontology (GO) and Kyoto Encyclopedia of Genes and Genomes (KEGG; https://www.genome.jp/kegg/) analysis. GO analysis includes three categories: 'Biological process' (BP), 'cellular component' (CC) and 'molecular function' (MF). Furthermore, a protein-protein interaction (PPI) network was also generated to demonstrate the association between these genes. PPI analysis was performed using STRING version 10.5 (https://string-db.org/). The results were visually presented using the ggplot2 package version 3.1.0 in $\mathrm{R}$ software (30). Providing that BRCA1-associated protein-1 (BAP1) mutation is a critical indicator for monitoring the poor progression of UM, the prognostic value of BAP1 mutation was determined in the present study. Data for UM patients, including BAP1 mutation status, were downloaded from the cBioportal database (http://www.cbioportal.org/).

\section{Results}

Survival-associated snoRNAs. After removing the low-abundance snoRNAs, 380 snoRNAs were submitted for survival analysis. Among these, 64 snoRNAs were significantly associated with the OS of patients with UM $(\mathrm{P}<0.05)$. The top 20 most significant survival-associated snoRNAs were presented in Fig. 1. Then, multivariate Cox analysis was performed and four snoRNAs were selected to construct a prognostic signature, including ACA45 (chr15: 83424696_83424823), ACA17 (chr9: 139621198_139621331), HBII-276 (chr8: 67834708_67834784) and SNORD12 (chr20: 47897219_47897309). A survival-risk formula was constructed based on the four snoRNAs as follows: ACA17 $*(-1.602)+\mathrm{ACA} 45 * 0.803+\mathrm{HBII}-276 * 0.603+$ SNORD12 * 1.348. Then, patients were divided into high- and low-risk groups according to median value of prognostic signature (Fig. 2). K-M survival analysis revealed that patients in the high-risk group exhibited shorter survival than those patients in the low-risk group (Fig. 3A). The number of patients in the high- and low-risk groups, and the number of censored patients at different time points are shown in Fig. 3B and C. ACA17 was significantly upregulated in the low-risk group, while ACA45, HBII-276 and SNORD12 were significantly upregulated in the high-risk group (Fig. 4). K-M survival plots were also used to present the prognostic value of each snoRNA (Fig. 5). ACA17 upregulation indicated better 
A

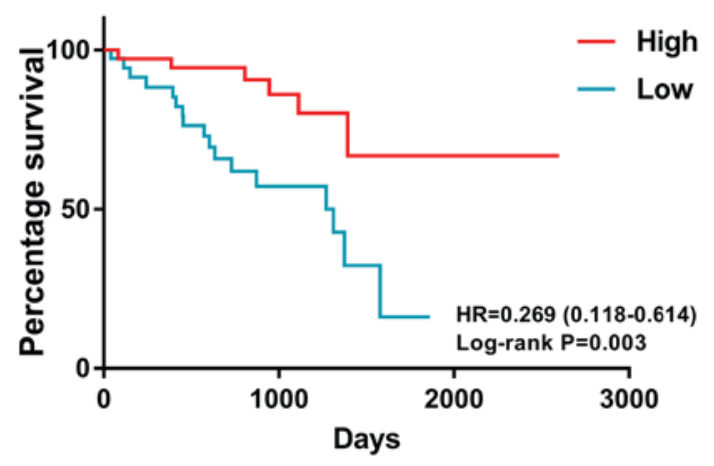

C

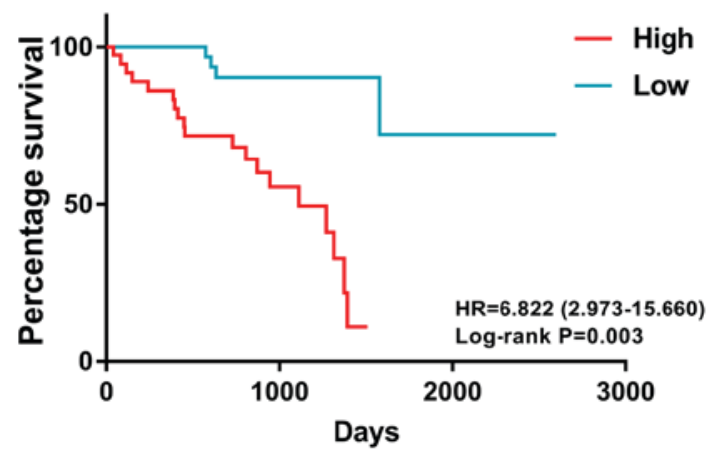

B

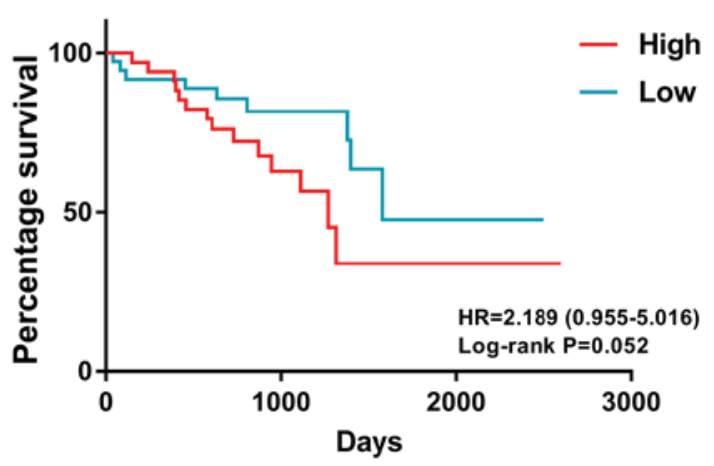

D

SNORD12

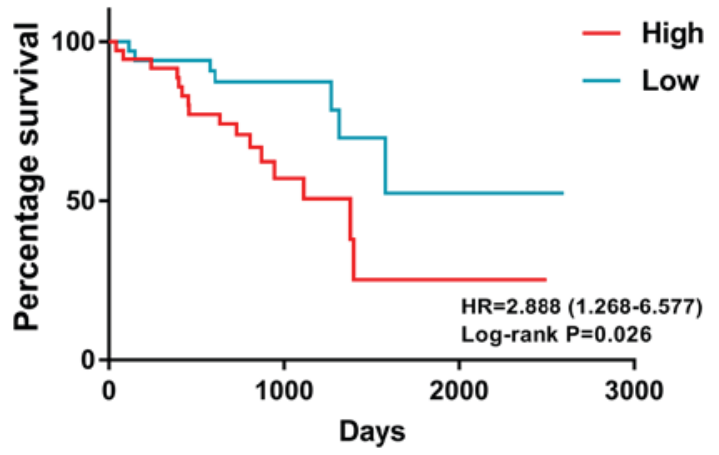

Figure 5. Prognostic value of the four small nucleolar RNAs. (A) Downregulated ACA17 indicated poor survival of patients with UM. Upregulated expression of (B) ACA45 (C) HBII-276 and (D) SNORD12 indicated poor survival of patients with UM. HR, hazard ratio; UM, uveal melanoma.

clinical outcome, while ACA45, HBII-276 and SNORD12 upregulation was associated with the poor survival of patients with UM.

Functional enrichment analysis. A total of 281 differentially expressed mRNAs between the high- and low-risk groups were determined (Fig. 6). PPI network analysis suggested that these genes notably interacted with each other (Fig. 7). Gene functional enrichment analysis revealed that these genes were significantly enriched in several biological processes and pathways. For BP, the most notably enriched functional terms were 'visual perception', 'regulation of rhodopsin-mediated signaling pathway' and 'rhodopsin-mediated signaling pathway' (Fig. 8A). Regarding CC, genes were markedly enriched in 'photoreceptor inner segment', 'photoreceptor disc membrane' and 'photoreceptor outer segment' (Fig. 8B). In regards to $\mathrm{MF}$, genes were notably enriched in 'structural constituent of eye lens', 'structural molecule activity' and 'hormone activity' (Fig. 8C). Importantly, KEGG enrichment analysis suggested that these genes were significantly associated with 'phototransduction', ' $\gamma$-aminobutyric acid (GABA) ergic synapse', 'amphetamine (APA) addiction', 'nicotine addiction' and 'neuroactive ligand-receptor interaction' (Fig. 9). Furthermore, the mutation status of BAP1 may be useful in predicting the clinical outcome of patients with UM; mutations in BAP1 demonstrated a significant association with poorer overall survival compared with UM patients without BAP1 mutations (Fig. 10A). Of note, the prognostic signature based on snoRNAs was markedly associated high-risk

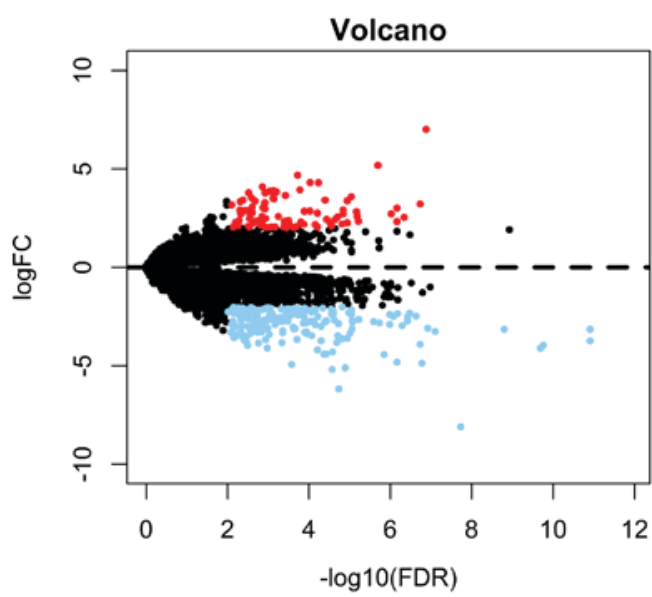

Figure 6. Volcano plot of the differentially expressed genes between the highand low-risk groups. Blue represents downregulated genes and red represents upregulated genes in the high-risk group.

patients with BAP1 mutations than those without in the low-risk group (Fig. 10B).

\section{Discussion}

In the present study, survival-associated snoRNAs in patients with UM were selected. Then, the snoRNAs that exhibited a significant prognostic value were determined and identified by multivariate analysis. Most importantly, a prognostic signature comprising four novel snoRNAs was proposed: ACA17, ACA45, HBII276 and SNORD12. Notably, the 


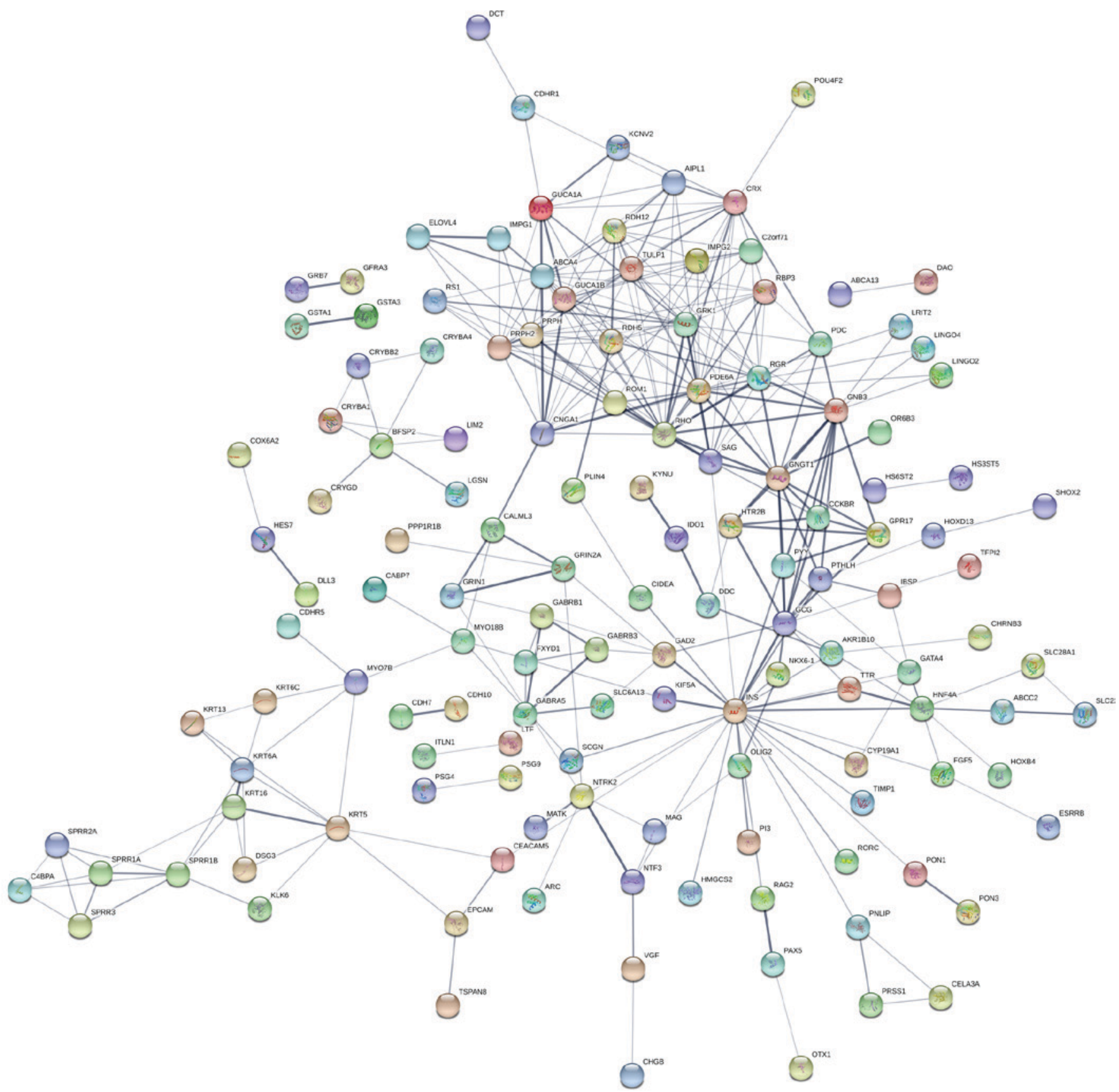

Figure 7. Protein-protein network demonstrating the interactions between differentially expressed genes.

prognostic signature of the present study, based on these four novel snoRNAs, may be an ideal risk model for patients with UM. To the best of our knowledge, the present study is the first to propose a prognostic signature based on snoRNAs. Furthermore, gene functional enrichment analysis revealed that the optic nerve-associated pathways are dysregulated most significantly between high-risk and low-risk groups. These findings may provide novel insight into the clinical management and molecular mechanisms underlying UM.

Previously, few studies have investigated the PI of UM from TCGA database. Xu et al (31) reported that high plasmacytoma variant translocation 1 (PVT1) expression was an independent predictor in patients with UM; it was inferred that the expression of PVT1 may act as a moderate and specific prognostic factor in terms of OS in UM. Wan et al (32) identified 21 co-expression modules that were analyzed based on 10,975 genes from $80 \mathrm{UM}$ samples, by using weighted correlation network analysis. It was revealed that the hub genes solute carrier family 17 member 7 , neurotrophic receptor tyrosine kinase 2, ankyrin repeat and BTB domain containing 1 and ADP-ribosylhydrolase like 1 may also serve a role as potential diagnostic and prognostic biomarkers of UM. Robertson et al (33) also analyzed the genomic data of 80 patients with UM; the patients were divided into several subgroups with different genomic aberrations, transcriptional features and clinical outcomes. Field et al (34) not only identified preferentially expressed antigen in melanoma (PRAME) expression as a biomarker for increased metastatic risk in class $1 \mathrm{UM}$; however, PRAME expression was associated with poor prognosis among class 2 UM cases (35). These studies provided novel and constructive 
A

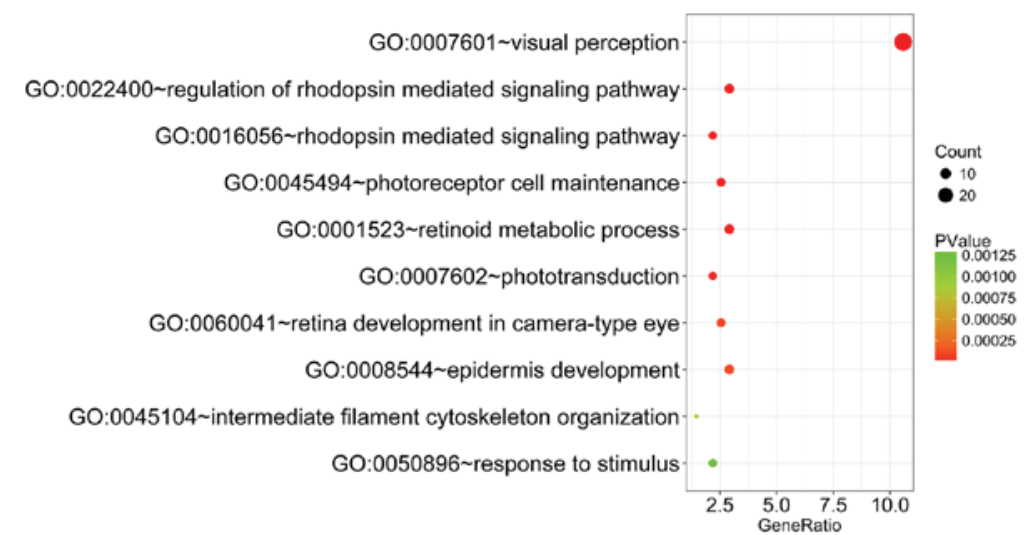

B

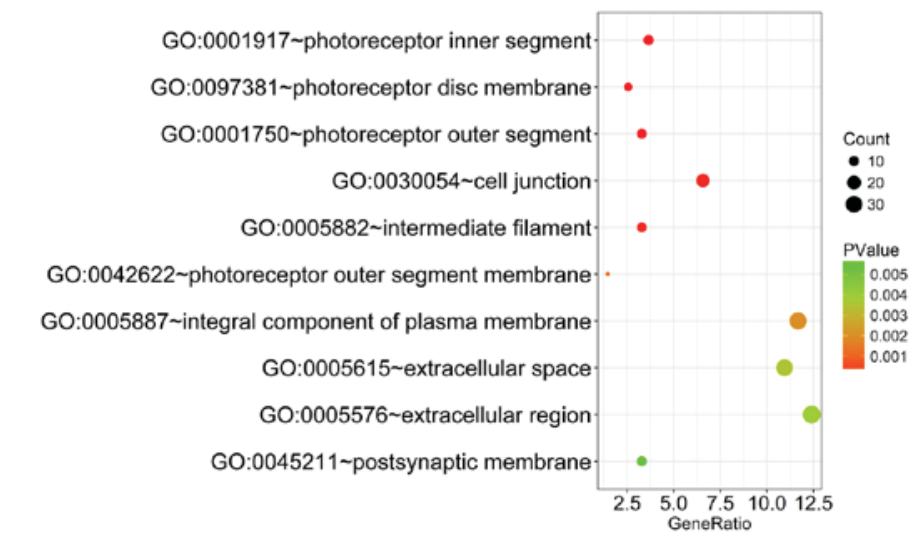

C

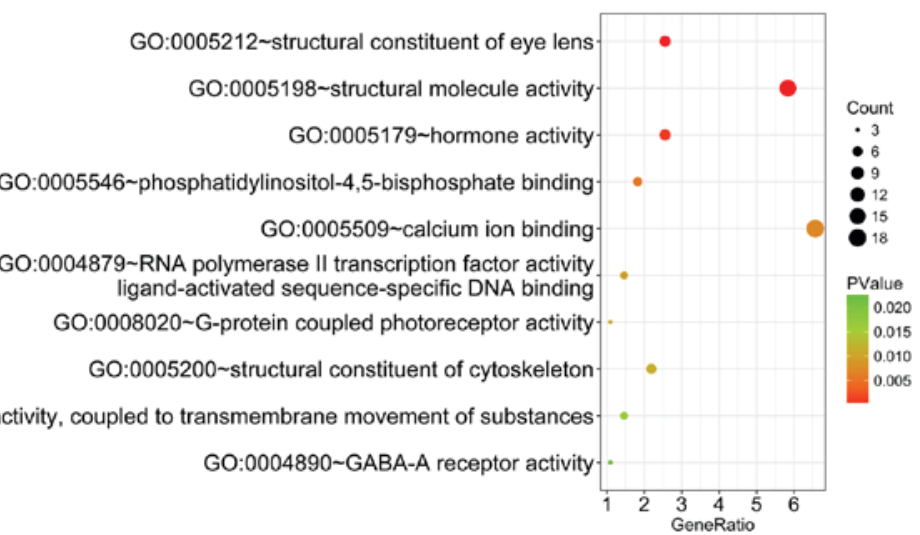

Figure 8. GO functional enrichment analysis of 283 common differentially expressed genes. Significantly enriched terms in the GO categories. (A) 'Biological process', (B) 'cellular component' and (C) 'molecular function'. GO, gene ontology.

methods of selecting prognostic biomarkers for UM; however, the role of snoRNAs in the prognosis of UM remains unclear. In the present study, a novel prognostic signature based on snoRNAs was developed. More importantly, the proposed risk score may attain satisfactory prognostic values for UM. With the advantage of high-throughput RNA-sequencing, numerous snoRNAs were identified; however, investigations into the clinical significance of snoRNAs in UM are limited. The present study may provide novel insight into the clinical management of UM.

Gene functional enrichment analysis revealed that several biological processes and pathways were associated with differentially expressed mRNAs between the high- and low-risk groups; however, the biological mechanisms underlying this association are unclear. The genes detected from KEGG enrichment analysis were demonstrated to be associated with 'phototransduction', 'GABAergic synapse' and 'APA addiction' in particular.
Brown etal (36) revealed that brain-modulated choroidal thickness has an unusual and well-established light dependence. In addition, Wicks et al (37) reported that ultraviolet phototransduction can evoke retina-dependent calcium flux in human epidermal melanocytes, and increased cellular melanin content. Furthermore, ocular melanocytosis is an important predisposing factor for UM (38). Therefore, it was speculated that phototransduction may be involved in the formation of UM in the present study. GABAergic synapses are important inhibitory neurotransmitters in the mammalian central nervous system (CNS), as they serve to hyperpolarize the postsynaptic neuron (39). Interestingly, 'APA addiction', 'nicotine addiction' and 'dopaminergic (DA) synapses' were also associated with UM; however, DA is an excitatory neurotransmitter in the CNS (40). APA promotes DA release in the CNS, whilst also inducing other biogenic amine-releasing and inhibitory neurons, and vesicle single-amine transporters (41). Monoamine oxidase can excite the CNS (42). 


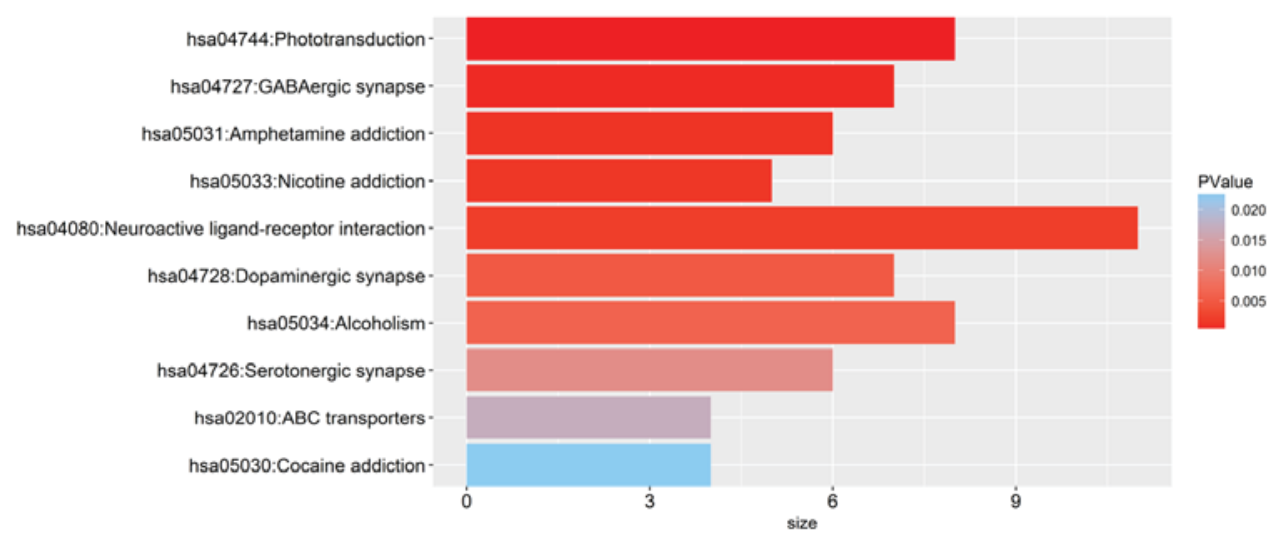

Figure 9. KEGG pathway analysis. The top 10 most significant KEGG pathways for differentially expressed genes. Size refers to the number of genes enriched in each pathway. Color indicates the P-value. KEGG, Kyoto Encyclopedia of Genes and Genomes.

A

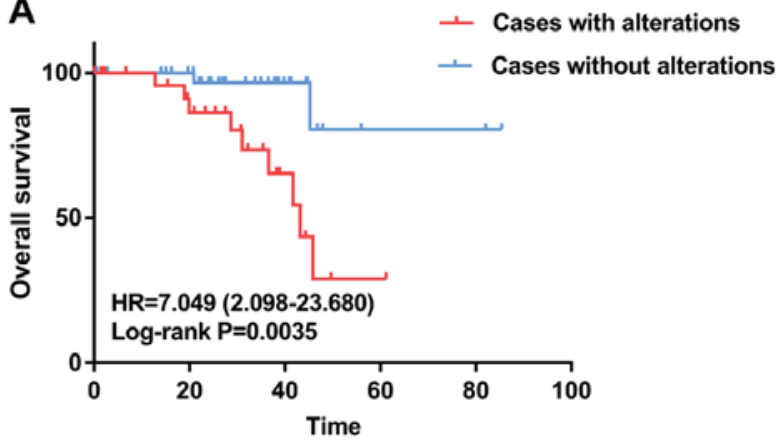

B

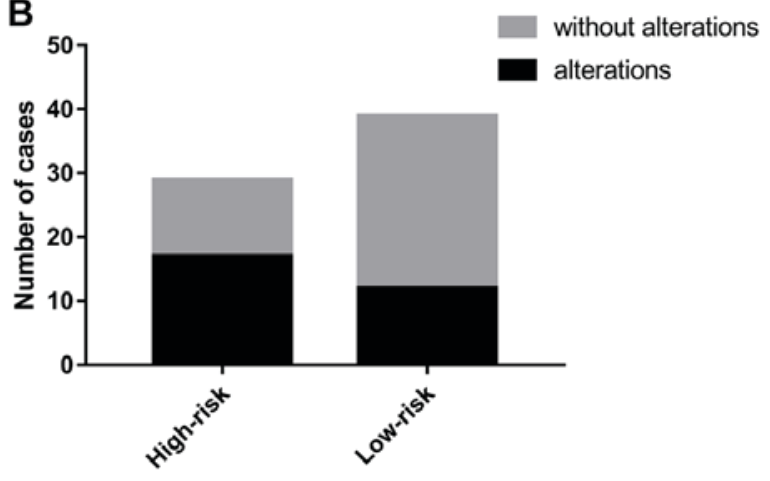

Figure 10. Association between BAP1 mutations and the small nucleolar RNA-based prognostic signature. (A) Patients with BAP1 mutation exhibited poor survival compared with patients without BAP1 mutation. (B) In the high-risk group, the number of patients with BAP1 mutation was greater than in low-risk group. BAP1, BRCA1-associated protein-1; HR, hazard ratio.

Nicotine initially inhibits DA release, which is then enhanced by regulating GABAergic neurons (43). This mechanism of action is similar to that of APAs in the CNS. Thus, it may be inferred that CNS activity-associated pathways may be notably dysregulated between high-risk and low-risk groups.

However, several limitations should be considered. An additional independent cohort was not used to validate the performance of prognostic signature. Furthermore, the in silico analysis for the molecular mechanisms require further investigation.

Collectively, the results of the present study demonstrated that snoRNAs may be notable prognostic markers for the survival of patients with UM. To the best of our knowledge, the present study is the first to demonstrate the prognostic value of snoRNAs, and provides novel insight into the complex biological functions underlying UM.

\section{Acknowledgments}

Not applicable.

\section{Funding}

The present study was supported by the National Natural Science Foundation of China (grant no. 81160119), a funding of Natural Science Foundation of Guangxi Zhuang
Autonomous Region (grant nos. 2017GXNSFAA198347 and 2016GXNsFAA380280).

\section{Availability of data and materials}

The datasets used and/or analyzed in the present study are available from the corresponding author on reasonable request.

\section{Authors' contributions}

W-JZ made substantial contributions to the design of the present study. QY and W-JZ conducted statistical analysis. QY wrote the manuscript which was revised by W-JZ. All authors read and approved the final manuscript.

\section{Ethics approval and consent to participate}

Not applicable.

\section{Patient consent for publication}

Not applicable.

\section{Competing interests}

The authors declare that they have no competing interests. 


\section{References}

1. Álvarez-Rodríguez B, Latorre A, Posch C and Somoza Á: Recent advances in uveal melanoma treatment. Med Res Rev 37: $1350-1372,2017$

2. Komatsubara KM, Manson DK and Carvajal RD: Selumetinib for the treatment of metastatic uveal melanoma: Past and future perspectives. Future Oncol 12: 1331-1344, 2016.

3. Reichstein D: New concepts in the molecular understanding of uveal melanoma. Curr Opin Ophthalmol 28: 219-227, 2017.

4. Vasalaki M, Fabian ID, Reddy MA, Cohen VM and Sagoo MS Ocular oncology: Advances in retinoblastoma, uveal melanoma and conjunctival melanoma. Br Med Bull 121: 107-119, 2017.

5. Kaliki S and Shields CL: Uveal melanoma: Relatively rare but deadly cancer. Eye (Lond) 31: 241-257, 2017.

6. Shields CL, Kels JG and Shields JA: Melanoma of the eye: Revealing hidden secrets, one at a time. Clin Dermatol 33 183-196, 2015

7. Helgadottir $\mathrm{H}$ and Höiom V: The genetics of uveal melanoma: Current insights. Appl Clin Genet 9: 147-155, 2016.

8. Heppt MV, Steeb T, Schlager JG, Rosumeck S, Dressler C, Ruzicka T, Nast A and Berking C: Immune checkpoint blockade for unresectable or metastatic uveal melanoma: A systematic review. Cancer Treat Rev 60: 44-52, 2017.

9. Blum ES, Yang J, Komatsubara KM and Carvajal RD: Clinical management of uveal and conjunctival melanoma. Oncology (Williston Park) 30: 29-32, 34-43, 48, 2016.

10. Krantz BA, Dave N, Komatsubara KM, Marr BP and Carvajal RD: Uveal melanoma: Epidemiology, etiology, and treatment of primary disease. Clin Ophthalmol 11: 279-289, 2017.

11. Zhou J, Jin B, Jin Y, Liu Y and Pan J: The antihelminthic drug niclosamide effectively inhibits the malignant phenotypes of uvea melanoma in vitro and in vivo. Theranostics 7: 1447-1462, 2017.

12. Cheng H, Chua V, Liao C, Purwin TJ, Terai M, Kageyama K Davies MA, Sato T and Aplin AE: Co-targeting HGF/cMET signaling with MEK inhibitors in metastatic uveal melanoma. Mol Cancer Ther 16: 516-528, 2017

13. Mahendraraj K, Lau CS, Lee I and Chamberlain RS: Trends in incidence, survival, and management of uveal melanoma: A population-based study of 7,516 patients from the surveillance, epidemiology, and end results database (1973-2012). Clin Ophthalmol 10: 2113-2119, 2016.

14. Andreoli MT, Mieler WF and Leiderman YI: Epidemiological trends in uveal melanoma. Br J Ophthalmol 99: 1550-1553, 2015.

15. Chandran SS, Somerville RPT, Yang JC, Sherry RM, Klebanoff CA, Goff SL, Wunderlich JR, Danforth DN, Zlott D, Paria BC, et al: Treatment of metastatic uveal melanoma with adoptive transfer of tumour-infiltrating lymphocytes: A single-centre, two-stage, single-arm, phase 2 study. Lancet Oncol 18: 792-802, 2017.

16. Komatsubara KM and Carvajal RD: Immunotherapy for the treatment of uveal melanoma: Current status and emerging therapies. Curr Oncol Rep 19: 45, 2017.

17. Spagnolo F, Picasso V, Spano L, Tanda E, Venzano C and Queirolo P: Update on metastatic uveal melanoma: Progress and challenges. BioDrugs 30: 161-172, 2016.

18. McMahon M, Contreras A and Ruggero D: Small RNAs with big implications: New insights into H/ACA snoRNA function and their role in human disease. Wiley Interdiscip Rev RNA 6: 173-189, 2015.

19. Zhou H, Xu Q, Ni C, Ye S, Xu X, Hu X, Jiang J, Hong Y, Huang D and Yang L: Prospects of noncoding RNAs in hepatocellular carcinoma. Biomed Res Int 2018: 6579436, 2018.

20. Koduru SV, Leberfinger AN and Ravnic DJ: Small non-coding RNA abundance in adrenocortical carcinoma: A footprint of a rare cancer. J Genomics 5: 99-118, 2017.

21. Siprashvili Z, Webster DE, Johnston D, Shenoy RM, Ungewickell AJ, Bhaduri A, Flockhart R, Zarnegar BJ, Che Y, Meschi F, et al: The noncoding RNAs SNORD50A and SNORD50B bind K-Ras and are recurrently deleted in human cancer. Nat Genet 48: 53-58, 2016.

22. Zhang Y, Xu C, Gu D, Wu M, Yan B, Xu Z, Wang Y and Liu H: H/ACA box small nucleolar RNA 7A promotes the self-renewal of human umbilical cord mesenchymal stem cells. Stem Cells 35 222-235, 2017

23. Huang C, Shi J, Guo Y, Huang W, Huang S, Ming S, Wu X, Zhang R, Ding J, Zhao W, et al: A snoRNA modulates mRNA $3^{\prime}$ end processing and regulates the expression of a subset of mRNAs. Nucleic Acids Res 45: 8647-8660, 2017.
24. Zhou F, Liu Y, Rohde C, Pauli C, Gerloff D, Köhn M, Misiak D, Bäumer N, Cui C, Göllner S, et al: AML1-ETO requires enhanced C/D box snoRNA/RNP formation to induce self-renewal and leukaemia. Nat Cell Biol 19: 844-855, 2017.

25. Xu B, Ye MH, Lv SG, Wang QX, Wu MJ, Xiao B, Kang CS and Zhu XG: SNORD47, a box C/D snoRNA, suppresses tumorigenesis in glioblastoma. Oncotarget 8: 43953-43966, 2017.

26. Patterson DG, Roberts JT, King VM, Houserova D, Barnhill EC, Crucello A, Polska CJ, Brantley LW, Kaufman GC, Nguyen M, et al: Human snoRNA-93 is processed into a microRNA-like RNA that promotes breast cancer cell invasion. NPJ Breast Cancer 3: 25, 2017

27. Yoshida K, Toden S, Weng W, Shigeyasu K, Miyoshi J, Turner J, Nagasaka T, Ma Y, Takayama T, Fujiwara T and Goel A: SNORA21-an oncogenic small nucleolar RNA, with a prognostic biomarker potential in human colorectal cancer. EBioMedicine 22: 68-77, 2017.

28. Xu L, Ziegelbauer J, Wang R, Wu WW, Shen RF, Juhl H, Zhang Y and Rosenberg A: Distinct profiles for mitochondrial t-RNAs and small nucleolar RNAs in locally invasive and metastatic colorectal cancer. Clin Cancer Res 22: 773-784, 2016.

29. Ritchie ME, Phipson B, Wu D, Hu Y, Law CW, Shi W and Smyth GK: limma powers differential expression analyses for RNA-sequencing and microarray studies. Nucleic Acids Res 43: e47, 2015 .

30. Wickham H: ggplot2: Elegant graphics for data analysis. Springer Publishing Company, Incorporated, 2009.

31. Xu H, Gong J and Liu H: High expression of lncRNA PVT1 independently predicts poor overall survival in patients with primary uveal melanoma. PLoS One 12: e0189675, 2017.

32. Wan Q, Tang J, Han Y and Wang D: Co-expression modules construction by WGCNA and identify potential prognostic markers of uveal melanoma. Exp Eye Res 166: 13-20,2018.

33. Robertson AG, Shih J, Yau C, Gibb EA, Oba J, Mungall KL, Hess JM, Uzunangelov V, Walter V, Danilova L, et al: Integrative analysis identifies four molecular and clinical subsets in uveal melanoma. Cancer Cell 32: 151, 2018.

34. Field MG, Decatur CL, Kurtenbach S, Gezgin G, van der Velden PA, Jager MJ, Kozak KN and Harbour JW: PRAME as an independent biomarker for metastasis in uveal melanoma. Clin Cancer Res 22: 1234-1242, 2016.

35. Field MG, Durante MA, Decatur CL, Tarlan B, Oelschlager KM, Stone JF, Kuznetsov J, Bowcock AM, Kurtenbach S and Harbour JW: Epigenetic reprogramming and aberrant expression of PRAME are associated with increased metastatic risk in Class 1 and Class 2 uveal melanomas. Oncotarget 7: 59209-59219, 2016.

36. Brown JS, Flitcroft DI, Ying GS, Francis EL, Schmid GF Quinn GE and Stone RA: In vivo human choroidal thickness measurements: Evidence for diurnal fluctuations. Invest Ophthalmol Vis Sci 50: 5-12, 2009.

37. Wicks NL, Chan JW, Najera JA, Ciriello JM and Oancea E: UVA phototransduction drives early melanin synthesis in human melanocytes. Curr Biol 21: 1906-1911, 2011.

38. Szuścik I, Romanowska-Dixon B, Jakubowska B and Orlowska-Heitzman J: Uveal melanoma in patients with ocular or oculodermal melanocytosis. Klin Oczna 110: 380-383, 2008 (In Polish).

39. Kuzirian MS and Paradis S: Emerging themes in GABAergic synapse development. Prog Neurobiol 95: 68-87, 2011.

40. Zieger E, Lacalli TC, Pestarino M, Schubert M and Candiani S: The origin of dopaminergic systems in chordate brains: Insights from amphioxus. Int J Dev Biol 61: 749-761, 2017.

41. Vicente-Rodríguez M, Rojo Gonzalez L, Gramage E, Fernández-Calle R, Chen Y, Pérez-García C, Ferrer-Alcón M, Uribarri M, Bailey A and Herradón G: Pleiotrophin overexpression regulates amphetamine-induced reward and striatal dopaminergic denervation without changing the expression of dopamine D1 and D2 receptors: Implications for neuroinflammation. Eur Neuropsychopharmacol 26: 1794-1805, 2016.

42. Baig AM: DARK side of amphetamine and analogues: pharmacology, syndromic manifestation, and management of amphetamine addiction. ACS Chem Neurosci 9: 2299-2303, 2018.

43. Ortells MO and Arias HR: Neuronal networks of nicotine addiction. Int J Biochem Cell Biol 42: 1931-1935, 2010. 